\title{
THE
}

\section{Dynamic Correlation Functions for the One-Dimensional $X Y Z$ Model: New Exact Results}

Gerhard Müller

University of Rhode Island, gmuller@uri.edu

Robert E. Shrock

Follow this and additional works at: https://digitalcommons.uri.edu/phys_facpubs

Terms of Use

All rights reserved under copyright.

\section{Citation/Publisher Attribution}

Gerhard Müller and Robert E. Shrock. Dynamic correlation functions for the one-dimensional XYZ model: new exact results. J. Magnetism and Magn. Materials 54-57 (1986), 1255-1256.

Available at: http://www.sciencedirect.com/science/article/pii/0304885386908061

This Article is brought to you for free and open access by the Physics at DigitalCommons@URI. It has been accepted for inclusion in Physics Faculty Publications by an authorized administrator of DigitalCommons@URI. For more information, please contact digitalcommons-group@uri.edu. 


\title{
Dynamic Correlation Functions for the One-Dimensional $X Y Z$ Model: New Exact Results
}

\author{
Gerhard Müller11 and Robert E. Shrock는 \\ 1 Department of Physics, University of Rhode Island, Kingston RI 02881, USA \\ 2 Institute for Theoretical Physics, State University of New York at Stony Brook, Stony Brook, NY 11974, USA
}

\begin{abstract}
It is found that there exist special circumstances for which a rigorous relation between the three dynamic structure factors $S_{\mu \mu}(q, \omega), \mu=x, y, z$, at $T=0$ of the one-dimensional spin- $s X Y Z$ model in a uniform magnetic field can be derived. This relation is used to infer new exact results for $S_{x x}(q, \omega)$ and $S_{y y}(q, \omega)$ of the $s=\frac{1}{2}$ anisotropic $X Y$ model.
\end{abstract}

We study the circumstances under which the general spin- $s X Z$ ferromagnet in a uniform magnetic field, specified by the Hamiltonian

$$
\mathcal{H}=-\sum_{l=1}^{N}\left[J_{x} S_{l}^{x} S_{l+1}^{x}+J_{y} S_{l}^{y} S_{l+1}^{y}+J_{z} S_{l}^{z} S_{l+1}^{z}+h S_{l}^{z}\right],
$$

for $J_{x}, J_{y}, J_{z} \geq 0$, even $N$ and periodic boundary conditions exhibits a ground state (GS) wavefunction of the simple product type (without loss of generality we also assume $J_{x} \geq J_{y}$ ):

$$
\begin{aligned}
|G\rangle & =\bigotimes_{l=1}^{N}|\Theta, l\rangle, \\
|\Theta, l\rangle & =U_{l}(\Theta)|s, l\rangle \\
& =\sum_{m=-s}^{s}|m, l\rangle D_{m s}^{(s)}(\cos \Theta / 2, \sin \Theta / 2) \\
& =\sum_{m=-s}^{s} \sqrt{\frac{(2 s) !}{(s+m) !(s-m) !}}(\cos \Theta / 2)^{s+m}(\sin \Theta / 2)^{s-m}|m, l\rangle .
\end{aligned}
$$

Here $U_{l}(\Theta)$ describes a unitary transformation representing a rotation of the spin direction at the site $l$ by an angle $\Theta$ away from the $z$-axis in the $x z$-plane, generated by the $(2 s+1)$-dimensional irreducible representation of the group $\mathrm{SU}(2)$ with matrix elements $D_{m s}^{(s)}$ as given above. For $\Theta \neq 0$ such a GS is characterized by the presence of spontaneous long-range order. The order parameter is

$$
\mathbf{M}=\left\langle\Theta, l\left|\mathbf{S}_{l}\right| \Theta, l\right\rangle=(s \sin \Theta, 0, s \cos \Theta) .
$$

There are evidently no correlated fluctuations in this state of maximum spin ordering.

The problem of finding special cases of the Hamiltonian $\mathcal{H}$ for which the GS wave function $|G\rangle$ has the form (2) is equivalent to finding the circumstances under which the Hamiltonian

$$
\tilde{\mathcal{H}}=U^{-1} \mathcal{H} U, \quad U=\bigotimes_{l=1}^{N} U_{l}(\Theta),
$$

has a GS wave function of the form

$$
|\tilde{G}\rangle=U^{-1}|G\rangle=\bigotimes_{l=1}^{N}|s, l\rangle,
$$


with all spins aligned parallel to the $z$-axis. The GS energy is invariant under this transformation:

$$
\langle G|\mathcal{H}| G\rangle=\langle\tilde{G}|\tilde{\mathcal{H}}| \tilde{G}\rangle=E_{G}
$$

The solution of this well-defined problem is that the $X Y Z$ model (1) does indeed have a GS wavefunction of the form (2) with [1]

$$
\cos \Theta=\sqrt{\left(J_{y}-J_{z}\right) /\left(J_{x}-J_{z}\right)},
$$

and energy

$$
E_{G}=-s^{2}\left(J_{x}+J_{y}-J_{z}\right),
$$

provided the exchange constants satisfy the constraints [2]

$$
J_{x} \geq J_{y} \geq J_{z},
$$

and the strength of the magnetic field is

$$
h=h_{N}=2 s \sqrt{\left(J_{x}-J_{z}\right)\left(J_{y}-J_{z}\right)} .
$$

The transformed Hamiltonian $\tilde{\mathcal{H}}$ whose GS wave function is $|\tilde{G}\rangle$ reads:

$$
\begin{aligned}
& \tilde{\mathcal{H}}=\sum_{l=1}^{N}\left\{J_{y}\left(S_{l}^{x} S_{l+1}^{x}+S_{l}^{y} S_{l+1}^{y}\right)+\left(J_{x}-J_{y}+J_{z}\right) S_{l}^{z} S_{l+1}^{z}+2 s\left(J_{y}-J_{z}\right) S_{l}^{z}\right. \\
& \left.+\sqrt{\left(J_{x}-J_{y}\right)\left(J_{y}-J_{z}\right)}\left[S_{l}^{z} S_{l+1}^{x}+S_{l}^{x} S_{l+1}^{z}-s\left(S_{l}^{x}+S_{l+1}^{x}\right)\right]\right\} .
\end{aligned}
$$

Note that the presence of a ferromagnetic GS does not guarantee that the ferromagnetic spinwave states are also eigenstates of $\mathcal{H}$ or $\tilde{\mathcal{H}}$. The spin-wave excitations with respect to the ferromagnetic state $|\tilde{G}\rangle$, for example, are characterized by the wave functions

$$
|\tilde{q}\rangle=S_{q}^{-}|\tilde{G}\rangle, \quad S_{q}^{-}=N^{-1 / 2} \sum_{l=1}^{N} \mathrm{e}^{-\mathrm{i} q l} S_{l}^{-} .
$$

The condition for these states to be eigenstates of $\tilde{\mathcal{H}}$ is that the second term on the right-hand side of the following equation vanishes:

$$
\left[\tilde{\mathcal{H}}, S_{q}^{-}\right]|\tilde{G}\rangle=\omega_{s w}(q)|\tilde{G}\rangle+\frac{1}{2} \sqrt{\left(J_{x}-J_{y}\right)\left(J_{y}-J_{z}\right)}\left(1+\mathrm{e}^{-\mathrm{i} q}\right) N^{-1 / 2} \sum_{l} \mathrm{e}^{-\mathrm{i} q l} S_{l}^{-} S_{l+1}^{-}|\tilde{G}\rangle
$$

where

$$
\omega_{s w}(q)=2 s\left(J_{x}-J_{y} \cos q\right)
$$

is the dispersion predicted by the linear spin-wave analysis. For general values of $J_{x}, J_{y}, J_{z}$ and $h$ satisfying the constraints (9) and (10), this condition is only met for $q=\pi$, for general $q$ only in the classical limit $s \rightarrow \infty$.

Thus the second term in (13) or, equivalently, the last term in (11) is responsible for nontrivial features in the $T=0$ dynamic structure factors defined as

$$
S_{\mu \nu}(q, \omega) \equiv \sum_{R} \mathrm{e}^{-\mathrm{i} q R} \int_{-\infty}^{+\infty} \mathrm{d} t \mathrm{e}^{\mathrm{i} \omega t}\left\langle S_{l}^{\mu}(t) S_{l+R}^{\nu}\right\rangle
$$

in spite of the very special structure of the GS wave function. However, the fact that $|\tilde{G}\rangle$ describes a state with all spins aligned in the $z$-direction implies the following general structure for the $S_{\mu \nu}(q, \omega)$ of $\tilde{\mathcal{H}}$ at $T=0$ :

$$
\begin{aligned}
S_{x x}(q, \omega)_{\tilde{\mathcal{H}}} & =S_{y y}(q, \omega)_{\tilde{\mathcal{H}}}=\frac{1}{4} S_{+-}(q, \omega)_{\tilde{\mathcal{H}}}, \\
S_{z z}(q, \omega)_{\tilde{\mathcal{H}}} & =4 \pi^{2} s^{2} \delta(q) \delta(\omega), \\
S_{\mu \nu}(q, \omega)_{\tilde{\mathcal{H}}} & =0 \quad \text { for } \quad \mu \neq \nu,
\end{aligned}
$$


where $S_{+-}(q, \omega)$ is the Fourier transform of $\left\langle S_{l}^{+}(t) S_{l+R}^{-}\right\rangle$and is, in general, nontrivial. This special structure is translated by the unitary transformation (4) into a relation between the three diagonal structure factors $S_{\mu \nu}(q, \omega)_{\mathcal{H}}$ of the $X Y Z$ model at $T=0$ and $h=h_{N}$. They are all expressible in terms of a single function, $S_{+-}(q, \omega)_{\tilde{\mathcal{H}}}$, as follows:

$$
\begin{aligned}
& S_{x x}(q, \omega)_{\mathcal{H}}=\frac{1}{4} S_{+-}(q, \omega)_{\tilde{\mathcal{H}}} \cos ^{2} \Theta+4 \pi^{2} s^{2} \sin ^{2} \Theta \delta(\omega) \delta(q), \\
& S_{y}(q, \omega)_{\mathcal{H}}=\frac{1}{4} S_{+-}(q, \omega)_{\tilde{\mathcal{H}}}, \\
& S_{z z}(q, \omega)_{\mathcal{H}}=\frac{1}{4} S_{+-}(q, \omega)_{\tilde{\mathcal{H}}} \sin ^{2} \Theta+4 \pi^{2} s^{2} \cos ^{2} \Theta \delta(\omega) \delta(q) .
\end{aligned}
$$

There exists a particular case of the $X Y Z$ model (1) for which these relations directly lead to new nontrivial exact results: the $s=1 / 2$ anisotropic $X Y$ model

$$
\mathcal{H}_{\gamma}=-J \sum_{l=1}^{N}\left[(1+\gamma) S_{l}^{x} S_{l+1}^{x}+(1-\gamma) S_{l}^{y} S_{l+1}^{y}\right]-h \sum_{l=1}^{N} S_{l}^{z} .
$$

For this model, which maps onto a system of noninteracting fermions via the Jordan-Wigner transformation [3], the dynamic correlation function $\left\langle S_{l}^{z}(t) S_{l+R}^{z}\right\rangle$ can be expressed as a fermion density-density correlation function [4]. The corresponding $T=0$ dynamic structure factor at $h=h_{N}=J \sqrt{1-\gamma^{2}}$ was recently determined in closed form [5]:

$$
\begin{aligned}
& S_{z z}(q, \omega)= \pi^{2} \frac{1-\gamma}{1+\gamma} \delta(q) \delta(\omega) \\
&+\frac{\gamma^{2}}{1-\gamma^{2}} \frac{\left[4 J^{2}\left(1-\gamma^{2}\right) \cos ^{2}(q / 2)-(\omega-2 J)^{2}\right]^{1 / 2}}{\left[\omega-2 J \sin ^{2}(q / 2)\right]^{2}+J^{2} \gamma^{2} \sin ^{2} q} \\
& \quad \times \Theta\left[4 J^{2}\left(1-\gamma^{2}\right) \cos ^{2}(q / 2)-(\omega-2 J)^{2}\right] .
\end{aligned}
$$

In contrast, the functions $\left\langle S_{l}^{x}(t) S_{l+R}^{x}\right\rangle$ and $\left\langle S_{l}^{y}(t) S_{l+R}^{y}\right\rangle$ are represented by infinite block Toeplitz determinants in the fermion language, i.e. quantities involving infinite products of fermion operators [6]. The spectrum of the corresponding $T=0$ dynamic structure factors $S_{x x}(q, \omega)$ and $S_{y y}(q, \omega)$ thus represent not just two-fermion excitations as is the case for $S_{z z}(q, \omega)$ but rather the excitation of $m$-fermion states with $m$ arbitrarily large. On the other hand, the newly found relations (17) imply that for $h=h_{N}$ all three dynamic structure factors are zero for values of $(q, \omega)$ outside the range of the two-particle spectrum, i.e. for $|\omega-2 J|>2 J h_{N} \cos (q / 2)$. They differ from one another (apart from the $\delta$-function at $q=\omega=0$ ) only by an overall $\gamma$-dependent factor [7].

These peculiar properties are far from evident in the formal expressions for $S_{x x}(q, \omega)$ and $S_{y y}(q, \omega)$ in the fermion representation. In fact, expressions (5.10) of ref. [6] which are stated to represent the two-particle contributions to $S_{x x}(q, \omega)$ are incompatible with our exact result unless one assumes that there are also contributions to these functions at $h=h_{N}$ from $m$-particle excitations with $m>2$. This would imply, however, that such contributions miraculously cancel one another for all $(q, \omega)$ outside the range of the two-particle spectrum.

Acknowledgment: The research of R.E.S. was supported in part by the National Science Foundation under the Grant PHY-81-9110-AOl. We have used a modified cmpj.sty style file.

\section{References}

1. As far as the ground-state properties are concerned, this analysis complements that reported by J. Kurmann, H. Thomas and G. Müller, Physica 112A (1982) 235, which focused on the XYZ antiferromagnet, but with a field in an arbitrary direction.

2. The constraint $J_{x} \geq J_{y}$ is just a convention. 
3. E. Lieb, T. Schultz and D. Mattis, Ann. Phys. (N.Y.) 16 (1961) 407; S. Katsura, Phys. Rev. 127 (1962) 1508;

4. T. Niemeijer, Physica 36 (1967) 377.

5. J.H. Taylor and G. Müller, Phys. Rev. B 28 (1983) 1529.

6. B.M. McCoy, E. Barouch and D.B. Abraham, Phys. Rev. A 4 (1971) 2331.

7. This result was already suspected in ref. [5] on the basis of sum rule considerations. 